Published in Diversité urbaine, 2018, vol. 18, p. 87-104

which should be cited to refer to this work.

\title{
La circulation des morts, l'ancrage des corps et le deuil sans frontières
}

\section{Circulation of the Dead, Anchoring of Bodies and Mourning without Borders}

\section{MARC-ANTOINE BERTHOD}

HES-SO, Haute école de travail social et de la santé, Lausanne, marc-antoine.berthod@eesp.ch

RÉSUMÉ - Lorsque la mort survient, l'attention se focalise sur la façon dont les collectivités fixent le devenir du cadavre. Il est plus rare de s'intéresser à la mobilité des morts, non seulement lorsqu'ils vont de leur lieu de décès à leur lieu de sépulture, mais aussi lorsqu'ils transitent d'un cimetière à un autre, lorsqu'ils sont exposés dans des lieux publics ou lorsqu'ils franchissent des frontières. S’appuyant sur une série de références en la matière et reprenant des éléments tirés d'une recherche menée auprès de familles migrantes ayant rapatrié un proche défunt, ce texte apporte quelques éclairages sur les formes que revêtent la mobilité des dépouilles et leurs ancrages, sur de brèves et de longues périodes, pour remettre en perspective certaines conceptions de la mort et du deuil.

ABSTRACT $\approx$ When death occurs, the focus is on how communities determine the destiny of the corpse. It is rarer for the mobility of the dead to be a concern; this includes not only the movement from where they died to their burial place, but also when they pass from one cemetery to another, when they are exposed in public places or when they cross borders. Based on documentary and field research conducted with migrant families who repatriated a deceased close relative, I seek to provide insight into the question of the forms of mobility of human remains and their anchorings, during short as well as long temporalities, so as to re-examine certain conceptions of death and mourning.

MOTS CLÉS = Mort, mobilité, corps, migration, deuil.

KEYWORDS - Death, mobility, bodies, migration, mourning. 


\section{Introduction}

Les morts circulent dans l'espace public plus souvent que l'on ne l'imagine. Transitant d'un point à un autre, les corps des personnes défuntes ou des parties de leur corps occupent une diversité de lieux, de manière plus ou moins temporaire, selon les motifs familiaux, politiques, administratifs, juridiques, techniques ou encore économiques qui organisent leur circulation. S'intéresser à ce phénomène permet de jeter un éclairage original sur les enjeux liés aux mobilités contemporaines et de remettre en perspective certaines conceptions de la mort et du deuil, en particulier celles qui postulent la nécessité de conduire un mort à sa dernière demeure, de fixer son statut et de le laisser en paix. Pour y parvenir, les réflexions qui suivent se nourrissent d'un ensemble de lectures, d'observations et d'entretiens menés dans le cadre de plusieurs recherches sur la fin de vie et sur le deuil. Elles prennent plus particulièrement appui sur une étude ethnographique portant sur le rapatriement des défunts en contexte migratoire ${ }^{1}$.

Ces réflexions empruntent un itinéraire en trois étapes. La première souligne l'importance d'observer les nombreuses situations lors desquelles les défunts, les cadavres, les corps, les cendres et les restes transitent d'un point à un autre. La deuxième met en évidence les sensibilités politiques qui sous-tendent la mobilité des morts et leur ancrage dans un lieu précis, notamment à travers les rituels. La troisième éclaire la tension qui existe entre ces composantes politiques et les conceptions personnelles et familiales relatives à cet ancrage. Suivant cet itinéraire, l'objectif est d'explorer les articulations entre la présence physique d'un cadavre et le sentiment d'être en présence d'un mort, en allant au-delà du seul registre funéraire.

\section{Les morts et les territoires}

L'historien Fustel de Coulanges a publié en 1864 son ouvrage le plus connu, La Cité antique (1984), dans lequel il analyse le fonctionnement de la Grèce et de Rome. Il s'applique à montrer comment ces sociétés se sont créées et modifiées avant de disparaître, en retraçant leur origine et 
leur histoire. Dans sa démonstration, il accorde une place centrale aux croyances qui seraient au fondement même de l'organisation sociale de ces deux entités politiques. De Coulanges soutient en effet que la mort était appréhendée comme un changement de vie et non pas comme une dissolution. Il en a découlé une nécessité de sépulture et de rituel, le culte des ancêtres, sans lesquels les vivants subiraient les représailles des défunts. La famille a été instituée et soutenue par une organisation sociale qui l'englobe afin d'assurer la production et la perpétuation du culte. En d'autres termes, c'est le tombeau familial, là où le mort est enterré, qui a induit le sentiment de propriété, régulé et garanti par la collectivité, celleci étant chargée de mettre en place les conditions favorables à la bonne tenue du culte des morts.

Si cette association entre famille, propriété et sépulture a été contestée dès la parution du livre et semble de nos jours rejetée - comme le note François Hartog dans sa préface à l'édition publiée chez Flammarion en 1984 -, je me contenterai d'apprécier et de commenter ici l'idée selon laquelle la mort et son ancrage dans un lieu précis constituent le moteur et le garant d'une importante sociabilité via le deuil. Pour Fustel de Coulanges, ce moteur de la sociabilité dépend étroitement des conceptions selon lesquelles les âmes vivaient littéralement sous terre et restaient dans la proximité des dépouilles. Dans la Cité antique, l'âme était comme enfermée avec le corps dans le tombeau. Fustel de Coulanges écrit à ce propos: «De cette croyance primitive dériva la nécessité de la sépulture. Pour que l'âme fût fixée dans cette demeure souterraine qui lui convenait pour sa seconde vie, il fallait que le corps, auquel elle restait attachée, fût recouvert de terre» (1984: 10). Autrement dit, sans tombeau, pas de demeure pour les défunts!

La perspective développée par Fustel de Coulanges fait de la croyance un élément qui précède et qui génère tout rituel. Cette idée, qu'il convient également de nuancer (Berthod 2015), a marqué bon nombre de travaux de sciences humaines, notamment des travaux ethnologiques, dès la moitié du $19^{\mathrm{e}}$ siècle. Elle se retrouve dans un ensemble de descriptions contemporaines postulant que les croyances guident les rituels funéraires, ces derniers étant interprétés comme le moyen mis collectivement en œuvre pour offrir aux morts leur dernière demeure sur la terre familiale d'origine, non seulement physiquement, mais aussi dans l'ordre des générations ou dans le monde des ancêtres.

Ce type d'approche part du principe selon lequel, dans les cas où les rituels ne sont pas effectués, les morts vont errer et hanter les vivants. Et, si les morts erraient dans des mondes souterrains dans la Grèce et la Rome antiques, bien d'autres formes d'errance ont été rapportées dans les collectivités d'ici et d'ailleurs, par le passé et jusqu'à nos jours. Il suffit de 
penser aux innombrables histoires de fantômes, de revenants et d'autres esprits qui attendent d'être fixés à leur dernière demeure pour trouver la paix, souvent sans que l'on sache ce qu'il est advenu du corps, ni même que l'on s'en préoccupe, d'ailleurs.

Faire reposer la réflexion sur le principe d'un nécessaire ancrage des morts à un lieu ou à un territoire induit la conséquence suivante: leur errance est considérée comme un risque. L'errance, qu'elle soit souterraine ou, de manière plus contemporaine, ubique, s'écarte de l'idéal de fixité et s'appréhende comme un problème à régler: il faudra y mettre un terme ${ }^{2}$. Ce risque d'errance des morts se pose avec d'autant plus d'acuité lorsque les individus sont inscrits dans des processus migratoires, car plusieurs ancrages territoriaux sont en concurrence. Le cas échéant, il peut devenir tentant de considérer que les rites d'enterrement, et les rapports aux morts dans leur ensemble, ne se déploient qu'en fonction des deux pôles qui détermineraient le «ici» et le «là-bas».

Ces pôles constitueraient alors l'alpha et l'oméga de l'articulation entre la migration, la mobilité, la mort et le deuil. C'est à partir d'eux que le risque d'errance des morts va être interprété et contrôlé. Dans cette perspective, le «ici» et le «là-bas», soit la terre d'origine et la terre d'accueil, s'apparentent à des points de référence allant de soi, à des lieux atemporels qui ne sont pas soumis à l'érosion du temps et aux changements. Brièvement dit, cela permet d'entretenir l'idée selon laquelle les morts sont appelés à reposer éternellement dans leur terre d'origine et, plus précisément, dans le tombeau familial, comme l'a suggéré Fustel de Coulanges.

Il est clair pourtant que les points d'arrivée et d'origine, de départ et d'installation sont eux-mêmes fugaces, hybrides et affectés par de multiples formes de mobilité (Chambers 1994; Clifford 1997; Farman 2012). Ils sont marqués par des successions de générations et des rituels qui ne sont jamais immuables. Il convient donc d'appliquer cette notion de fluidité et de mobilité aussi aux rapports que les vivants entretiennent avec les morts, ce qui implique de ne pas centrer l'attention sur deux points fixes non questionnables, souvent incarnés par les funérailles pratiquées « ici » et celles qui ont lieu «là-bas».

Pour apprécier l'articulation entre la mort, la migration et le deuil, il importe au contraire de prendre en considération les dynamiques qui se laissent observer non seulement dans tous les «entre-deux», mais aussi, et surtout, en deçà et au-delà des rituels qui seraient propres aux divers pôles de départ et d'arrivée des migrants, dans la multiplicité des sphères sociales et de la vie quotidienne (Berthod 2016). Autrement dit, il convient d'inverser la perspective et de mettre en évidence la circulation des morts, sur de longues périodes, parfois, pour apprécier à leur juste mesure 
l'hybridité et l'inventivité des pratiques individuelles et sociales qui la sous-tendent, dans l'ensemble des lieux, les nécrogéographies, ou deathscapes, pour reprendre l'expression anglaise, où s'inscrivent et se déploient ces pratiques ${ }^{3}$.

Pour illustrer cette inversion, évoquons brièvement l'exemple de Dracula, le roman de l'écrivain irlandais Bram Stoker (2003), qui s'inscrit dans le même esprit du temps que l'ouvrage de Fustel de Coulanges. Je ne pense pas ici aux nombreuses déclinaisons cinématographiques de ce personnage, mais au livre original qui est, parmi de nombreuses autres interprétations possibles, un excellent témoignage de la naissance de la psychiatrie, de l'accompagnement en fin de vie et des actions à entreprendre pour donner un statut au mort et pour l'attacher à un territoire avec un arrière-plan de migration.

Pour tuer un vampire, et en particulier le comte Dracula, qui se déplace en permanence (à noter que le titre prévu du livre était encore, quelques semaines avant son impression, en mai 1897, The Un-dead, soit le «non-mort»), il convient de lui couper la tête et de ficher un pieu dans sa poitrine. Le point intéressant avec ce geste tient moins dans la volonté assassine qui le motive que dans la nécessité de transpercer le corps avec le pieu pour le ficher dans la terre. Stoker signale, en effet, que le cercueil qui transporte le corps de Dracula est recouvert d'une couche de sa terre d'origine; le pieu doit s'y planter.

Ce détail déterminant rappelle l'importance de l'articulation entre la terre d'origine, le cadavre et la conception de la personne qui le transcende. Il est produit ici de manière fort singulière, car cette articulation ne s'opère pas en fonction de deux pôles fixes, mais entre divers éléments mobiles et syncrétiques, là où se trouve le défunt. Une telle articulation apparaît alors comme une solution en transit, clairement détachée du sentiment de propriété que Fustel de Coulanges mettait de l'avant. L'exemple de Dracula permet d'associer la circulation des corps et des morts à l'espace public dans des contextes qui outrepassent le temps et l'espace des rites funéraires. Il met l'accent sur le fait que la question de la mort et du deuil, et le rapport à tel mort en particulier, s'ouvre et se ferme à de nombreuses occasions, en fonction de contextes singuliers, et jamais de manière définitive.

Dans un autre registre, les archéologues Andrew Chamberlain et Michael Pearson (2001) partent justement de cette perspective inversée. Ils rappellent que la plupart des gens pensent que les corps sont traités avec dignité et respect et que les morts reposent définitivement en paix. Or, quiconque a eu l'occasion d'assister au changement d'affectation d'un cimetière sait que tel n'est pas le cas: les cercueils, les corps et les squelettes, comme le relèvent ces deux archéologues, sont passés au bulldozer 
et déplacés. Après plusieurs années, la plupart des restes humains sont relocalisés ou incinérés; les dépouilles ne reposent jamais pour l'éternité. Leurs destins sont par ailleurs très diversifiés. Pour s'en rendre compte, il suffit de lire le livre de la journaliste Mary Roach (2003) consacré au devenir des cadavres. Ces derniers se retrouvent chez les étudiants en médecine, dans des crash tests ou dans des expérimentations scientifiques sur la décomposition. Ils sont aussi sollicités pour des transplantations ou encore embaumés, voire répartis en plusieurs lieux, après avoir été réduits en cendres.

Si l'utilisation des dépouilles, des restes ou des parties de corps se décline de multiples façons, elle demeure toutefois confinée à des espaces peu accessibles au public (Robben 2004). Il est rare de voir de «vrais cadavres», ce qui peut laisser à penser que les morts ne circulent pas ou peu. Il arrive néanmoins que les morts soient transportés et exhibés dans des espaces accessibles au grand public; pensons aux momies présentées dans les musées et aux reliques dans les églises. La mise en scène des corps «plastinés» par l'anatomiste allemand Günther von Hagens dans de grandes salles d'exposition est certainement l'exemple le plus connu du grand public ${ }^{4}$. Est-il possible de parler d'errance des morts quand leurs corps et des parties de leurs corps circulent dans plusieurs pays? Et qu'advient-il du risque de leur errance quand le rapport au cadavre semble coupé du deuil? Ces exemples mettent en évidence le fait que les processus migratoires ne sont qu'une facette de la circulation et de la présence des morts dans les espaces publics et privés. Ils montrent que leur souvenir ne saurait être complètement dissocié de cette mobilité des corps, ni uniquement déterminé par l'idée d'un lieu de sépulture définitif et clairement situé, deux aspects allant souvent de pair.

\section{Les sensibilités politiques de la mobilité post-mortem}

Ces constats conduisent à l'étape suivante de mon itinéraire, dont l'objectif est de faire ressortir les sensibilités politiques qui conditionnent cette circulation physique des morts et son articulation au deuil. Il convient de noter à cet égard que l'exposition des corps, ou leur simple présence dans des espaces accessibles à un public soit de professionnels, soit de curieux, est toujours susceptible de monstration excessive - pensons aux théâtres anatomiques à la fin du 19e siècle (Sappol 2004) - et d'offuscation - pensons au scandale qui a suivi la découverte en France de 351 foetus dans la chambre mortuaire de l'hôpital Saint-Vincent-dePaul en 2006 (Wolf 2012).

Si les États administrent cette circulation des corps et des parties de corps ainsi que leur mise en scène (Esquerre et Truc 2011), il convient 
d'aller au-delà des dimensions juridique, administrative et technique pour comprendre ces deux réactions. La circulation des morts recèle une force politique susceptible de déterminer son articulation au deuil, en particulier dans les situations de crise, lors de catastrophes naturelles ou d'épidémies et quand les décès surviennent en masse à la suite de migrations forcées ou de guerres. La présentation des corps morts et leur mise en circulation témoignent autant des enjeux de pouvoir qui prévalent au sein d'une collectivité que des excès de pouvoir dont une administration peut faire preuve. Comme le souligne Valérie Souffron (2015: 14): «qu'ils les abandonnent ou qu'ils les encadrent, les États gouvernent les corps et tentent de les maîtriser. Ce qui ne va pas sans poser problème. Si le cadavre est politique, c'est aussi par ce qu'il pourrait incarner».

Le fait de posséder et de faire circuler des reliques permet d'éclairer cette composante politique rattachée aux fragments de dépouilles. Entre le $9^{\mathrm{e}}$ et le $11^{\mathrm{e}}$ siècle, le vol et le déplacement de reliques étaient fréquents, comme l'a décrit Patrick Geary (1993). Cet historien rappelle que la relique n'est pourtant rien sans son contexte: il faut lui attribuer des valeurs, en l'occurrence la guérison ou les vertus chrétiennes. Il s'agit d'attacher des récits aux morts et de les faire parler. La dimension politique se loge dans cette association entre le corps ou la partie de corps, ce qui en est dit ou montré, et les personnes qui parlent en leur nom et les font circuler.

Un millénaire plus tard, la question des reliques reste d'actualité. En 2013, l'archéologue Nevzat Çevik s'est par exemple adressé au Vatican pour réclamer le retour des ossements de saint Nicolas - évêque de Myre, mort en 345, dont les restes ont été pillés en 1087 puis dispersés et entreposés à Bari en Italie, à Fribourg en Suisse et à Saint-Nicolas-de-Port en France - dans sa ville d'origine en Turquie. Relayée par la presse, cette information n'a pas plu au prévôt de la cathédrale de Fribourg, qui jurait qu'il n'allait jamais restituer les reliques de saint Nicolas.

Parmi de nombreux autres, cet exemple atteste de la fragilité des modes de gouvernance des corps et en particulier des corps morts visant à articuler la présence physique du cadavre (ou une partie seulement) à un contexte social significatif, dont la mise en scène est susceptible d'être disputée sur de très longues périodes par diverses instances qui vont au-delà des appareils administratifs et étatiques. Cette sensibilité politique des corps morts et de leur circulation oscille entre exhibition et offuscation au nom d'une autorité et d'une légitimité potentiellement contestables.

À l'instar des reliques, les corps morts font en effet l'objet de nombreuses revendications. Les réenterrements de personnalités politiques ou religieuses après l'effondrement de l'ancien bloc socialiste de l'Europe de l'Est, au tournant des années 1990, en sont emblématiques. L'anthropologue Katherine Verdery a montré combien «la matérialité d'un corps 
peut être déterminante pour son efficacité symbolique» (1999: 27, ma traduction). Si les corps ont l'avantage de la concrétude, leur signification a toutefois moins à voir avec celle-ci qu'avec la façon dont les gens pensent à leur propos: «Les morts arrivent avec un curriculum vitae» (ibid.: 28, ma traduction), sans pouvoir parler en leur propre nom.

Se demandant pourquoi le corps mort comme symbole provoque tant d'émotions, Verdery suggère que les restes humains comportent une dimension identificatoire et affective, dans la mesure où chacun possède un corps. Elle soutient de surcroît que la composante politique des corps morts s'intrique avec des représentations collectives plus larges, d'ordre transcendantal. Dans cette perspective, les corps morts apparaissent comme «d'excellents moyens pour accumuler des éléments essentiels aux changements politiques: le capital symbolique. Les corps morts, en résumé, peuvent faire l'objet d'un profit politique» (ibid.: 33, ma traduction).

Autrement dit, réclamer le rapatriement d'un corps permet d'affirmer ou de combattre des identités nationales, voire religieuses. Ceux qui le font estiment que ces «trésors culturels», ossements, cendres, corps momifiés, requièrent un enterrement digne et décent au nom d'une nation ou d'une patrie ${ }^{6}$, indépendamment des chroniques familiales susceptibles de tisser le souvenir de la personne défunte. Ils cherchent à affirmer, sinon à redessiner, des zones d'appartenance et contribuent à façonner ainsi les liens sociaux. Les réenterrements analysés par Verdery ne servent donc pas qu'à légitimer les nouvelles politiques postcommunistes: ils remodèlent des visions du monde.

L'usage de ces corps et leur circulation relèvent de processus anthropologiques qui touchent à la moralité d'une communauté, aux changements spatio-temporels et à la définition des liens entre les individus et les groupes: «Du début à la fin du livre, mes arguments s'intéressent à la façon dont nous pouvons penser la légitimité politique, moins en termes rationalistes et plus adéquatement en termes "cosmiques", pour montrer les processus riches, complexes et controversés de la politique comme action créatrice de sens, elle-même alimentée et animée par ce sens » (ibid.: 52, ma traduction). Citant Fustel de Coulanges, l'anthropologue estime que, si la présence des morts consacrait le lien entre lieu d'enterrement et système de parenté dans la Cité antique, elle institue un lien similaire avec l'idée de la nation.

Or, ces réflexions s'appliquent aux morts qui viennent avec un «curriculum vitae». Restent-elles pertinentes quand cet usage concerne des corps anonymes? Le cas échéant, les revendications de réenterrer ces morts, ou ne serait-ce déjà que de les enterrer, surviennent là où l'appartenance à une terre est ambiguë, impossible à déterminer, voire rejetée, 
et là où le lieu de repos des corps est considéré comme impropre et, de ce fait, nécessairement temporaire. Dans ces contextes, la sensibilité politique inscrite au cœur de l'articulation entre le lieu d'inhumation, le territoire et le deuil ne disparaît pas.

Elle se dévoile, au contraire, avec d'autant plus de force que la mort vient briser, souvent de manière tragique, un parcours migratoire. Alexandra Délano Alonso et Benjamin Nienass (2016) ont édité à ce sujet le numéro de la revue Social Research intitulé «Borders and the Politics of Mourning». Les différentes contributions qui le constituent montrent que le deuil des migrants disparus et inconnus peut être porté autant par des citoyens que par les familles. Les démarches visant à retrouver les corps et à les identifier afin de les enterrer dignement et, dans la mesure du possible, de les remettre à leurs proches et de les rapatrier sont analysées comme des actes de résistance aux limites concrètes des Étatsnations. Quand elles sont médiatisées, ces démarches tendent à désigner les responsabilités, tout particulièrement celles des administrations. Par conséquent, elles servent aussi à repenser le lien social.

Les performances réalisées par le collectif berlinois du Center for Political Beauty ${ }^{7}$ sont révélatrices de ces démarches. Face aux nombreuses personnes tragiquement décédées en tentant de franchir l'une des frontières de l'Union européenne, les membres de ce collectif ont entrepris d'aller chercher des corps de victimes pour les enterrer dans la capitale allemande. Leur action, nommée Les morts arrivent et largement médiatisée, ambitionne d'offrir à ceux qui n'ont pas pu atteindre leur destination de le faire après leur mort. Une telle action est non seulement motivée par la volonté d'humaniser les tombes de fortune, parfois avec l'aide des familles, et d'enterrer dignement ces morts après les avoir identifiés, mais aussi de sensibiliser l'opinion publique sur la façon dont l'État gère le rapport à ces derniers, entre monstration excessive et offuscation.

Ainsi, en juin 2015, une mère syrienne décédée trois mois plus tôt en Méditerranée et enterrée dans un cimetière sicilien avec l'inscription «Inconnue $\mathrm{n}^{\circ} 2$ » a été exhumée et identifiée, puis transportée dans un cimetière berlinois par ce collectif. Elle a bénéficié de funérailles en présence d'une centaine de citoyens et de militants, d'officiants religieux et de journalistes. On avait disposé parmi eux des chaises vides portant les noms des membres du gouvernement, invités à la cérémonie. Celle-ci s'est déroulée avec l'aval du mari, qui a suivi les funérailles grâce à un lien vidéo, car il n'était pas autorisé à se déplacer librement dans le pays en tant que requérant d'asile ${ }^{8}$.

Présenté comme une performance, Les morts arrivent est une forme de «civisme forensique», ce qui correspond aux actions entreprises par des ensembles de citoyens engagés, des proches de disparus et des 
organismes associatifs ou étatiques liés à la prise en charge des corps et à l'anthropologie médico-légale (Schwartz-Marin et Cruz-Santiago 2016). Créant des bases de données au sein des communautés migrantes, exhumant les corps qui bordent les frontières pour les identifier, les remettre à leurs familles et les enterrer dignement, ces groupes, parfois faiblement institués, témoignent de cette sensibilité politique qui caractérise la circulation des morts et qui configure ce lien entre les corps, le lieu d'inhumation et le territoire.

Ces actions rappellent par ailleurs que le deuil n'est pas qu'une affaire individuelle et psychologique; c'est aussi une affaire collective et politique et il n'appartient pas qu'aux familles. Face aux restes d'un inconnu, le deuil devient acte de résistance pour que justice soit faite. C'est pour cette raison que certains proches de migrants s'opposent parfois à la tenue de funérailles officielles ou de cérémonies commémoratives, initiées même en l'absence des corps. Le cas échéant, la ritualisation induirait un sentiment de clôture du deuil alors que les proches attendent des éclaircissements sur une disparition ou sur une mort. Autrement dit, la présence des corps inhumés dans un territoire qui ne leur correspond pas devient un levier pour revendiquer une plus grande justice sociale et refonder les liens sociaux et communautaires à partir de la vulnérabilité que chacun ressent dans ces situations (Butler 2004).

\section{Les familles migrantes et les deuils sans frontières}

De nombreuses initiatives citoyennes et politiques réclament l'inhumation des morts dans un lieu bien déterminé. Elles contribuent à faire du deuil une force susceptible de redéfinir ou de refonder le lien social lorsque le défunt est une figure publique éminente et lorsque le corps a disparu ou ne peut être identifié. Ces situations restent cependant rares. La plupart des morts qui transitent le font discrètement, dans des espaces transfrontaliers et dans des temporalités souvent plus brèves que dans les exemples évoqués jusqu'à présent. Que se passe-t-il quand les morts ne font l'objet d'aucune revendication, hormis celle qu'auraient formulée le défunt lui-même de son vivant ou ses proches? Le cas échéant, comment se tisse ce lien entre le territoire, la présence des corps et le deuil ? Et comment se positionnent les proches dès lors que différentes conceptions de la nation ou de la patrie entrent en jeu? La dernière partie de ce texte apporte quelques réponses à ces questions, en prenant appui sur les migrations transnationales, quand le corps est bel et bien présent et que la mort ne produit pas de sentiment d'injustice particulier.

L'intérêt de se focaliser sur les migrations transnationales tient dans le fait que cette articulation entre le territoire, la mort et la conception 
de la personne ressort avec acuité, dans la mesure où une telle articulation s'institue dans des réseaux interétatiques souvent bien identifiables et analysables (Basch et al. 1994), ce qui est particulièrement le cas de la migration portoricaine aux États-Unis à laquelle je me réfère ici (Duany 2002; Grosfoguel 2003, ainsi que la note 1 pour une description du contexte de la recherche que j'ai menée). La question de la mort au sein de ces réseaux et les enjeux qu'elle soulève auprès des personnes, des familles et des collectivités ont d'ailleurs fait l'objet d'une attention grandissante ces dernières années (Koskinen-Koivisto et al., à paraître).

Pour mon argument, il convient de signaler d'emblée que les points de vue des personnes décédées et des membres de leur famille ne correspondent pas toujours aux revendications politiques, citoyennes, nationalistes, religieuses, voire éducatives, scientifiques et artistiques ou encore militaires ${ }^{9}$ des personnes impliquées dans la circulation des morts et dans la proclamation d'un lieu idoine d'inhumation (Berthod 2006b). Si la volonté des défunts et celle de leur famille se superposent avec ces revendications collectives, pour autant qu'elles existent, elles s'en distinguent également. En se concentrant sur les personnes concernées par leur mort ou par celle d'un proche, et en analysant leurs actions, leurs motivations ou leurs justifications du choix du lieu d'inhumation et plus largement leur deuil, d'autres conceptions apparaissent.

Pour ces sujets, les pôles de «départ» et d'«arrivée», comme cela a été évoqué auparavant, sont toujours plus flous, instables et changeants que ne le laissent entendre les appels à un rapatriement. Leurs contours fluctuent au fil des parcours de vie et les personnes en ont bien conscience ${ }^{10}$. Cette fugacité des points d'origine et des points d'arrivée mérite d'être prise en considération quand la question de la mort s'imbrique à celle de la migration, comme l'a bien décrit Milan Kundera (2003). Dans son roman L'ignorance, l'écrivain rappelle que le terme «nostalgie»vient du grec nostos, qui signifie «retour», et d'algos, qui signifie «mal» ou «douleur». La nostalgie s'apparente à la souffrance causée par le désir inassouvi de rentrer dans son pays, dans sa patrie, dans sa nation, chez soi. Kundera attire l'attention sur le terme espagnol añorar qui signifie «avoir de la nostalgie» et qui dérive du latin ignorare, soit «ignorer». Ici, la nostalgie est non seulement associée à la souffrance de l'absence et de l'éloignement, mais surtout à l'ignorance des chroniques familiales, culturelles et sociales qui se trament sur les lieux mêmes que l'on a quittés. La nostalgie est plus que la souffrance de la rupture des liens : elle est la crainte de l'oubli. Elle est la crainte d'oublier et de se faire oublier tout à la fois.

Kundera dépeint la façon dont ce sentiment tragique d'ignorance émerge, avec force et violence, à travers le personnage de Josef qui, après 
plus de vingt ans d'exil, retourne pour la première fois dans sa ville natale de Prague. Dès son arrivée, Josef se rend dans un cimetière qu'il ne trouve pas tout de suite: la topographie s'est transformée depuis son départ. Quand il arrive enfin sur la tombe de ses parents, il mesure toute l'ignorance liée à son absence, en voyant la liste des personnes ajoutées sur la pierre tombale: «Il regarda la stèle; le marbre était couvert de nombreux noms : apparemment, la tombe était devenue, entre-deux, un grand dortoir» (2003: 61). Josef visualise les corps posés sur celui de sa mère, qu'il avait autrefois mis en terre. Il reste songeur en lisant les noms des personnes qu'il ne connaissait pas. Mais il est surtout frappé par la lecture des noms de celles qu'il connaissait, car il n'avait pas été averti de leur mort. À cet instant, ce défaut d'information devient le signe d'un oubli mutuel, le signe d'une ignorance qui traduit d'un seul coup le fossé temporel qui caractérise l'éloignement, la migration et l'exil.

La lecture de ce roman m’a été suggérée par Helena, une Portoricaine trentenaire établie à New York depuis cinq ans, lorsque j'ai mené un entretien avec elle en 2004 sur son parcours migratoire et sur sa conception des rapatriements posthumes. En se référant à Kundera, Helena souligne toute l'importance que revêt un tel sentiment d'étrangeté dans ses propres questionnements identitaires. Ce sentiment résulte de la distance temporelle que chacun ressent à l'égard de son parcours de vie, marqué par les déplacements et par les éloignements. À l'instar du personnage de Josef, Helena estime que le temps estompe autant sa représentation des relations familiales et interpersonnelles que sa représentation des lieux où elle a longtemps vécu, comme San Juan, la capitale de Porto Rico, avant de s'établir aux États-Unis. C'est la façon dont ces deux types de représentations se transforment au gré de l'expérience migratoire qui motivera le choix de son lieu d'inhumation.

L'écart entre le «maintenant» et le «auparavant» imprègne l'expérience intime de la migration. Il dépasse et englobe l'opposition entre le «ici» et le «là-bas». Cet écart temporel se révèle lorsqu'il s'agit d'être confronté à sa propre mort ou à celle d'un proche ${ }^{11}$. Les personnes qui ne sont plus retournées dans leur pays d'origine depuis plusieurs décennies le ressentent avec force; elles craignent d'être désillusionnées en cas de retour sur les lieux qu'elles ont quittés jadis. C'est le cas de Clara, qui était âgée de 82 ans lors de notre rencontre et qui réside dans un établissement médico-social pour personnes âgées dans le quartier de East Harlem. Elle a vécu plus de 60 ans à New York sans jamais être retournée à Porto Rico, une île dont elle ne conserve que des impressions fugaces, souvent idéalisées et même stéréotypées, selon sa propre perception. Elle n'y a d'ailleurs plus de famille: ses enfants, nés aux États-Unis, se sont installés dans diverses villes du pays. Si Clara souhaite reposer dans le cimetière 
de son village natal, elle sait que son rapatriement posthume dépendra des coûts que cela engendrera, de la disponibilité des emplacements dans le cimetière et de la décision de ses enfants.

Ces considérations pragmatiques paramètrent le choix du lieu d'inhumation. Car encore faut-il, dans les cas où l'argent ne constitue pas un obstacle $^{12}$, pouvoir disposer d'un emplacement dans le caveau familial ou à proximité de la tombe d'un parent - tout le monde ne peut pas reposer auprès du même être cher - ou tout simplement être autorisé à reposer dans le cimetière de son choix si l'on ne réside pas dans la commune qui l'administre. C'est pourquoi celui qui souhaite se faire rapatrier va anticiper et contrôler son destin posthume en achetant une tombe à l'avance, voire en payant déjà les frais de transport. Mais cela ne constitue pas toujours une garantie suffisante. Venu comme jeune adulte à New York, Pedro, à présent cinquantenaire, avait entrepris de telles démarches pour reposer auprès de sa mère, décédée à Porto Rico quelques années plus tôt. Or, il a dû céder son emplacement à l'une de ses tantes résidant dans l'île et décédée dans l'intervalle. C'est cette dernière qui repose désormais aux côtés de sa propre sœur; ce ne sera pas lui.

Selon l'âge du défunt, l'implication des familles dans le choix du lieu d'inhumation change. Une personne jeune, sans famille, qui décède accidentellement ou des suites d'une maladie verra les probabilités de se faire rapatrier augmenter considérablement par rapport aux personnes plus âgées (Berthod 2006b). Et le fait d'avoir soi-même un conjoint et surtout des enfants s'avère déterminant. Ces derniers peuvent ne pas prendre en considération le souhait du défunt d'être inhumé dans le lieu de son choix, non seulement pour des raisons liées aux frais occasionnés par le rapatriement et par le déplacement des proches pour suivre le corps et les cérémonies, mais aussi pour des questions pratiques de proximité entre le lieu d'inhumation et leur propre lieu de vie. La décision des proches de rapatrier ou non un corps leur incombe d'autant plus que la volonté du défunt n'a jamais été clairement exprimée ni répétée, ce qui est le cas dans de nombreuses familles.

Et parmi les personnes qui manifestent leur volonté de se faire rapatrier $^{13}$, il est intéressant de noter que la dimension nationaliste et patriotique, si elle est fréquemment mentionnée et avérée dans bon nombre d'activités quotidiennes des Portoricains (Duany 2002; Flores 2000), constitue rarement la principale raison. C'est le rapport nostalgique entretenu avec un lieu et un environnement apprécié quelques années auparavant, situé parfois ailleurs, «là-bas», qui ressort comme l'élément le plus significatif chez les personnes que j'ai rencontrées. Pour Hector, ce sont les souvenirs d'une enfance heureuse et insouciante à Porto Rico qui alimentent son désir de retour posthume; pour Otilio, c'est l'attachement 
à un paysage qu'il a autrefois connu et admiré, mais qu'il sait considérablement transformé depuis son départ.

Ces quelques exemples montrent que la définition du lieu de destination posthume s'articule à un imaginaire qui repose sur une base existentielle individuelle (Berthod 2007). La définition de ce lieu est néanmoins disputée quand le défunt est une personnalité publique, quand le décès génère un sentiment d'injustice, voire d'indignité. L'écart et surtout l'appréciation de cet écart entre un «maintenant» et un "auparavant» jouent donc un rôle important dans l'articulation entre la mort et le deuil dans les contextes migratoires, à l'instar de ce qu'a relevé Balkan (2015).

Il en résulte que le choix du lieu d'inhumation ne représente pas ici l'aboutissement d'une trajectoire migratoire ni le sommet du sentiment tragique de la vie immigrée, comme l'avance par exemple Yassine Chaïb dans Lémigré et la mort (2000) à propos des rapatriements des défunts Tunisiens depuis la France. Ce choix ne vise pas non plus à correspondre étroitement aux conceptions de la «bonne mort» ni aux croyances qui seraient propres au pays d'origine et nécessiteraient la mise en œuvre de rituels funéraires «authentiques", trop souvent associés à des pratiques culturelles immuables qui seraient distribuées de manière homogène au sein d'une collectivité donnée.

Le choix du lieu d'inhumation indique plutôt l'ampleur de cet écart temporel pour la personne qui se projette dans son destin posthume et pour les proches qui rapatrient le corps, au-delà des frontières étatiques, nationales ou idéologiques qui s'y combinent et qui cristallisent en certaines circonstances l'opposition, obligeant à des négociations (Hunter 2016), entre un «ici» et un «là-bas». Ce choix révèle la façon dont les individus s'approprient un territoire et se positionnent par rapport à leurs délimitations tant légales que symboliques. Au fond, il constitue moins un enjeu national que citoyen, car il dénote des processus d'appartenance et des modes de participation à la vie collective dépassant les seuls critères légaux de résidence et d'assignation à un État (Berthod, à paraître).

De plus, il est à noter que les expériences vécues antérieurement par les individus face à la mort d'un proche et leurs perceptions du traitement du cadavre, que ce soit inscrit ou non dans des processus migratoires, peuvent revêtir plus d'importance que la difficulté à respecter la conformité d'un rituel funéraire, du moins en dehors des contextes musulmans qui sont souvent étudiés en la matière. Le simple fait d'exprimer une volonté de se faire rapatrier comporte le risque d'être très concrètement exposé à l'absence réelle de parentèle, de liens ou de ressources sur le lieu de destination posthume et de révéler au grand jour cette forme d'ignorance dépeinte par Kundera (2003). 
Il convient, par conséquent, d'intégrer plus systématiquement ces éléments à la réflexion pour appréhender le rapport que les communautés migrantes entretiennent avec leurs morts. Il s'agit pour cela d'aller au-delà de la seule temporalité des funérailles dans les analyses, en considérant notamment la période de deuil (Rachédi et al. 2016). Il importe d'observer aussi les multiples actions individuelles et collectives entreprises dans d'autres sphères sociales: comment les migrants appréhendent-ils leur fin de vie, la plupart du temps dans des établissements médicalisés? Ou, comment vivent-ils la mort et le deuil d'un proche sur leur lieu de travail, dans leurs cercles d'amis ou dans l'intimité de leur vie quotidienne? S'intéresser à l'histoire des parcours migratoires permet enfin de saisir la façon dont chaque individu a vécu une ou des confrontations avec la mort au fil de ses expériences et de comprendre ainsi l'impact que ces confrontations ont sur le rapport concret à la mort. Cela permet de saisir également la façon dont les normes et pratiques collectives sont vécues, négociées et transformées, tout particulièrement dans les réseaux transnationaux.

C'est en combinant ces éléments qu'il devient possible de comprendre la façon dont l'articulation entre la circulation des morts, le deuil et l'ancrage à la terre déploie sa composante politique et caractérise la dynamique des liens sociaux qui se refondent, se recréent ou s'effacent dans une multiplicité de contextes (Berthod 2018, 2016). Cela peut se faire sans nécessairement postuler l'existence d'un système de croyances homogènes, comme le suggérait Fustel de Coulanges, ni d'une représentation sociale de la «bonne mort» qui guiderait a priori les pratiques autour de la mort et du deuil et qui s'incarnerait tout particulièrement dans les funérailles.

\section{Notes}

1. Intitulée Les territoires de la mort. Étude anthropologique des enjeux culturels vécus par les familles portoricaines établies à New York face à la mort, cette recherche a été subventionnée par le Fonds national suisse de la recherche scientifique et réalisée entre octobre 2003 et septembre 2005. Des observations ont été menées au sein d'entreprises de pompes funèbres et lors de funérailles, à New York et à Porto Rico. Une cinquantaine d'entretiens approfondis ont été conduits avec des Portoricains de première, deuxième ou troisième générations établis à New York ainsi qu'avec des professionnels de l'accompagnement des personnes en fin de vie, des travailleurs sociaux, des employés de pompes funèbres, des responsables de morgues et de cimetières. Pour des détails sur le contexte politique particulier de Porto Rico et les mouvements migratoires entre cette île et le continent qui ne seront pas abordés ici, je me permets de renvoyer le lecteur à de précédents articles (Berthod 2007, 2006a, 2006b).

2. La problématique de l'errance n'est pas spécifique aux morts; elle fait miroir à celle que rencontrent les «exilés» ou les «réfugiés» (Malkki 1992) et à la façon dont la 
mobilité des migrants, souvent fragmentée et non linéaire, reste perçue et conceptualisée (Collyer 2007).

3. Une sous-discipline a émergé pour analyser ces deathscapes: la nécrogéographie, soit l'étude des pratiques d'enterrement, des lieux où reposent les corps et de leur configuration ainsi que de l'inscription de la mémoire des défunts dans le paysage et dans l'espace public (Marjavaara 2012).

4. Voir: www.bodyworlds.com [consulté le $1^{\text {er }}$ octobre 2018].

5. Voir l'article de Yelmarc Roulet publié le 9 janvier 2013 dans le quotidien Le Temps, «Frisson pour la relique de saint Nicolas», https://www.letemps.ch/suisse/2013/01/09/ frisson-relique-saint-nicolas [consulté le $1^{\text {er }}$ octobre 2018].

6. C'est notamment le cas du chef Sioux Long Wolf, rapatrié d'un cimetière londonien en septembre 1997 après y avoir reposé une centaine d'années, afin d'être enterré auprès de ses ancêtres dans le Dakota du Sud. Pour plus de détails à ce propos et sur les enjeux éthiques de l'archéologie funéraire en général, voir Williams et Giles (2016).

7. The Center for Political Beauty is an assault team that establishes moral beauty, political poetry and human greatness while aiming to preserve humanitarianism. http://politicalbeauty.com [consulté le $1^{\text {er }}$ octobre 2018]. Pour un commentaire critique sur leurs performances, voir l'article de von Bieberstein et Evren (2016).

8. Voir l'article de Melissa Eddy publié le 16 juin 2015 dans le New York Times: «Migrant's Funeral in Berlin Highlights Europe's Refugee Crisis». https://www. nytimes.com/2015/06/17/world/europe/migrants-funeral-in-berlin-highlightseuropes-refugee-crisis.html?_r=1 [consulté le $1^{\text {er }}$ octobre 2018].

9. Si ces différents points méritent une attention précise, je ne fais que les évoquer ici, dans la mesure où ils concernent moins directement les processus migratoires.

10. Sans explicitement traiter de la mort ou du deuil à la suite d'un décès, l'ouvrage édité par André Aciman (1999) reflète très bien cette imbrication entre la permanence, la mémoire et la fugacité à travers le point de vue personnel de cinq écrivains qui commentent leur propre expérience de l'exil. Pour de plus amples développements sur ces imbrications, voir Eng et Kazanjian (2003).

11. Ce point se révèle aussi dans le rapport que les individus entretiennent avec leur propre corps au fil du temps. Eduardo, affecté par le sida, a migré de Porto Rico à New York pour bénéficier de meilleurs soins. Durant notre entretien, il souligne vouloir être incinéré car il ne supporte plus de voir son corps se dégrader. Pour Eduardo, c'est ce rapport de soi à soi qui conditionne son approche de la mort. L'éventualité de se faire inhumer à Porto Rico, une île qu'il ne souhaitait pas quitter, lui apparaît comme secondaire, notamment du fait que son homosexualité déclarée n'a pas été acceptée par tous ses proches qui y vivent encore.

12. À noter que les rapatriements des défunts constituent désormais des niches commerciales bien développées (Lestage 2012).

13. D’après le registre démographique du Département de la santé de Porto Rico, il y a eu 76379 rapatriements dans cette île entre les années 1951 et 2003. Selon les chiffres de l'office des statistiques de la ville de New York, le nombre de défunts retournés depuis cette ville vers Porto Rico correspondait à 14,5\% environ de tous les décès des membres de cette communauté au début des années 2000. Ce pourcentage était quasiment identique entre les personnes décédées nées à Porto Rico et celles nées à New York de parents migrants. 


\section{Bibliographie}

Aciman, A. (dir.), 1999. Letters of Transit. Reflections on Exile, Identity, Language, and Loss. New York, The New Press.

Balkan, O., 2015. «Burial and Belonging», Studies in Ethnicity and Nationalism, vol. 15, $\mathrm{n}^{\mathrm{o}}$ 1, p. $120-134$.

Basch, L., N. Glick Schiller et C. Szanton Blanc, 1994. Nations Unbound. Transnational Projects, Postcolonial Predicaments and Deterritorialized Nation-States. Luxembourg, Gordon \& Breach Publishers.

Berthod, M.-A., à paraître. «Mort, territoires et citoyenneté», in G. Clavandier et F. Michaud-Nérard (dir.), Que vont devenir les cimetières? Paris, Hermann.

Berthod, M.-A., 2018. «Le deuil», in A. Piette et J.-M. Salanskis (dir.), Dictionnaire de l'Humain. Paris, Presses universitaires de Paris Nanterre, p. 101-108.

Berthod, M.-A., 2016. «Le paysage relationnel du deuil», Frontières, vol. 26, n 1-2. https://www.erudit.org/fr/revues/fr/2014-v26-n1-2-fr02300/1034383ar/ [consulté le $1^{\text {er }}$ octobre 2018].

Berthod, M.-A., 2015. «L'indiscipline conceptuelle des rites: enjeux interprétatifs en thanatologie», in D. Jeffrey et A. Cardita (dir.), La fabrication des rites. Québec, Presses de l'Université Laval, p. 29-48.

Berthod, M.-A., 2007. «Juan Sánchez, un artiste engagé en quête d'identité et de mémoire», Ethnographiques.org, n 13. http://www.ethnographiques.org/2007/ Berthod_2 [consulté le 11 avril 2017].

Berthod, M.-A., 2006a. «De si beaux cadavres : réflexions sur les soins de conservation des morts», L'autre. Cliniques, cultures et sociétés, vol. 7, n 3, p. 427-440.

Berthod, M.-A., 2006b. «Expérience migratoire et identité dans la mort transnationale: les défunts portoricains rapatriés de New York», Canadian Journal of Latin American and Caribbean Studies / Revue canadienne des études latino-américaines et des Caraïbes, vol. 61, n 31, p. 145-168.

Butler, J., 2004. Precarious Lives. The Power of Mourning and Violence. Londres, Verso.

Chaïb, Y., 2000. L'émigré et la mort. La mort musulmane en France. Aix-en-Provence, Édisud.

Chamberlain, A. et M. Pearson, 2001. Earthly Remains. The History and Science of Preserved Human Bodies. Londres, The British Museum Press.

Chambers, I., 1994. Migrancy, Culture, Identity. Londres, Routledge.

Clifford, J., 1997. Routes. Travel and Translation in the Late Twentieth-Century. Cambridge, Harvard University Press.

Collyer, M., 2007. «In-Between Places: Trans-Saharan Transit Migrants in Morocco and the Fragmented Journey to Europe ", Antipode. A Radical Journal of Geography, vol. 39, no 4, p. 668-690.

De Coulanges, F., 1984 [1864]. La Cité antique. Paris, Flammarion.

Délano, A. et B. Nienass, 2016. «Introduction: Borders and the Politics of Mourning», Social Research, vol. 83, n 2, p. XIX-XXXI.

Duany, J., 2002. The Puerto Rican Nation on the Move. Identities on the Island and in the United States. Chapel Hill, University of North Carolina Press.

Eng, D. L. et D. Kazanjian (dir.), 2003. Loss. The Politics of Mourning. Berkeley, University of California Press. 
Esquerre, A. et G. Truc, 2011. «Les morts, leurs lieux et leurs liens», Raisons politiques, $n^{\circ} 41 / 1$, p. 5-11.

Farman, A., 2012. Clerks of the Passage. Westmount, Linda Leith Publishing.

Flores, J., 2000. From Bomba to Hip-Hop. Puerto Rican Culture and Latino Identity. New York, Columbia University Press.

Geary, P., 1993. Le vol des reliques au Moyen Âge: furta sacra. Paris, Aubier.

Grosfoguel, R., 2003. Colonial Subjects. Puerto Ricans in a Global Perspective. Berkeley, University of California Press.

Hunter, A., 2016. «Deathscapes in Diaspora: Contesting Space and Negotiating Home in Contexts of Post-Migration Diversity», Social \& Cultural Geography, vol. 17, n² 2, p. $247-261$.

Koskinen-Koivisto, E., S. Saramo et H. Snellman (dir.), à paraître. Transnational Death. Helsinki, Finnish Literature Society.

Kundera, M., 2003. L'Ignorance. Paris, Gallimard.

Lestage, F., 2012. «Entre Mexique et États-Unis: la chaîne entrepreneuriale de la mort et des migrants», Revue Européenne des Migrations Internationales, vol. 28, nº 3, p. 71-88.

Malkki, L., 1992. «National Geographic: The Rooting of Peoples and the Territorialization of National Identity among Scholars and Refugees», Cultural Anthropology, vol. 7, n 1 , p. 22-44.

Marjavaara, R., 2012. «The final Trip. Post-Mortal Mobility in Sweden», Mortality, vol. $17, \mathrm{n}^{\circ}$ 3, p. 256-275.

Rachédi, L., C. Montgomery et B. Halsouet, 2016. «Mort et deuil en contexte migratoire: spécificités, réseaux et entraide», Enfances, Familles, Générations, n 24. http://www.erudit.org/fr/revues/efg/2016-n24-efg02827/1038114ar/ [consulté le $1^{\text {er }}$ octobre 2018].

Roach, M., 2003. Stiff. The Curious Life of Human Cadavers. New York, W. W. Norton \& Company.

Robben, A. (dir.), 2004. Death Mourning and Burial: A Cross-Cultural Reader. Malden, Blackwell Publishing.

Sappol, M., 2004. A Traffic of Dead Bodies. Anatomy and Embodied Social Identity in Nineteenth-Century America. Princeton, Princeton University Press.

Schwartz-Marin, E. et A. Cruz-Santiago, 2016. «Pure Corpses, Dangerous Citizens: Transgressing the Boundaries between Experts and Mourners in the Search for Disappeared in Mexico ", Social Research, vol. 83, n 2, p. 483-510.

Souffron, V., 2015. «Chairs disparues», Communications, n 97, p. 7-15.

Stoker, B., 2003 [1897]. Dracula. Londres, Penguin Classics.

Verdery, K., 1999. The Political Lives of Dead Bodies. Reburial and Postsocialist Change. New York, Columbia University Press.

Von Bieberstein, A. et E. Evren, 2016. «From Aggressive Humanism to Improper Mourning: Burying the Victims of Europe's Border Regime in Berlin», Social Research, vol. 83, n 2, p. 453-479.

Williams, H. et M. Giles (dir.), 2016. Archeologists and the Dead. Mortuary Archeology in Contemporary Society. Oxford, Oxford University Press.

Wolf, J., 2012. «Le travail en chambre mortuaire: invisibilité et gestion en huis clos», Sociologie du travail, vol. 54, n 2, p. 157-177. 